\title{
A factorisation of diffusion measure and finite sample path
}

\section{constructions}

\author{
Alexandros Beskos, Omiros Papaspiliopoulos ${ }^{\dagger}$ and Gareth O. Roberts ${ }^{\ddagger}$
}

May 24, 2007

\begin{abstract}
In this paper we introduce decompositions of diffusion measure which are used to construct an algorithm for the exact simulation of diffusion sample paths and of diffusion hitting times of smooth boundaries. We consider general classes of scalar timeinhomogeneous diffusions and certain classes of multivariate diffusions. The methodology presented in this paper is based on a novel construction of the Brownian bridge with known range for its extrema, which is of interest on its own right.
\end{abstract}

Keywords : Rejection sampling, exact simulation, conditioned Brownian motion, boundary hitting times.

\section{Introduction}

In this paper, we shall give explicit and a.s. finite constructions for diffusion processes. Our methods give rise to efficient algorithms which can simulate exactly (i.e. free of any time discretisation error and subject only to finite computer precision) from any finite-dimensional distribution of the diffusion. Moreover, we devise an algorithm for the simulation of diffusion hitting times of smooth boundaries. The methodology presented in this paper is based on a novel construction of the Brownian bridge with known range for its extrema.

\footnotetext{
${ }^{*}$ Mathematics Institute, University of Warwick, a.beskos@warwick.ac.uk

${ }^{\dagger}$ Department of Statistics, University of Warwick, o.papaspiliopoulos@warwick.ac.uk

${ }^{\ddagger}$ Department of Mathematics and Statistics, Lancaster University, g.o.roberts@lancaster.ac.uk
} 
For ease of exposition, we commence by considering the one-dimensional diffusion process $X=\left\{X_{s} ; s \in[0, t]\right\}$ defined through the Stochastic Differential Equation (SDE):

$$
\mathrm{d} X_{s}=\alpha\left(X_{s}\right) \mathrm{d} s+\mathrm{d} B_{s}, \quad X_{0}=x, s \in[0, t]
$$

where $B=\left\{B_{s} ; s \in[0, t]\right\}$ is a scalar Brownian motion and $\alpha: \mathbf{R} \mapsto \mathbf{R}$ some drift function. In later sections we will discuss generalizations to time-inhomogeneous and multivariate processes.

Assume that (1) admits a unique weak and non-explosive solution. Let $\mathbb{Q}$ and $\mathbb{W}$ denote the law of $X$ and the Wiener measure respectively on $[0, t]$ for the initial condition $X_{0}=B_{0}=x$. In the rest of the paper we assume the following conditions.

$\left(\mathrm{C}_{0}\right)$ The Radon-Nikodym derivative of $\mathbb{Q}$ w.r.t. $\mathbb{W}$ exists and it is given by Girsanov's formula,

$$
\frac{\mathrm{d} \mathbb{Q}}{\mathrm{d} \mathbb{W}}=\exp \left\{\int_{0}^{t} \alpha\left(X_{s}\right) \mathrm{d} X_{s}-\int_{0}^{t} \frac{1}{2} \alpha^{2}\left(X_{s}\right) \mathrm{d} s\right\}
$$

$\left(\mathrm{C}_{1}\right) \alpha \in C^{1}$,

$\left(\mathrm{C}_{2}\right) \alpha^{2}+\alpha^{\prime}$ is bounded below.

The aim is to perform rejection sampling from $\mathbb{Q}$ using candidates from $\mathbb{W}$. Under $\left(\mathrm{C}_{1}\right)$, we can use Itô's lemma to rewrite Girsanov's formula as

$$
\frac{\mathrm{d} \mathbb{Q}}{\mathrm{d} \mathbb{W}}=\exp \left\{A\left(X_{t}\right)-A(x)-\int_{0}^{t} \frac{\alpha^{2}\left(X_{s}\right)+\alpha^{\prime}\left(X_{s}\right)}{2} \mathrm{~d} s\right\},
$$

for

$$
A(u)=\int_{0}^{u} \alpha(z) \mathrm{d} z, \quad u \in \mathbf{R} .
$$

The theory to be presented extends the work of Beskos et al. (2006) which provides simulation algorithms under the additional condition:

$\left(^{*}\right) \lim \sup _{u \rightarrow \infty}\left(\alpha^{2}+\alpha^{\prime}\right)(u)<\infty$, or $\lim \sup _{u \rightarrow-\infty}\left(\alpha^{2}+\alpha^{\prime}\right)(u)<\infty$.

Whilst $\left(\mathrm{C}_{0}\right)-\left(\mathrm{C}_{2}\right)$ will be naturally satisfied in many contexts, $\left({ }^{*}\right)$ is certainly restrictive (for example is not met in the Ornstein-Uhlenbeck case). In this paper we remove $\left(^{*}\right)$. With small modifications, the resulted algorithm can also provide exact draws from the 
distribution of the hitting time of one-sided smooth boundaries for the Brownian motion and other diffusions. In comparison with our previous work in Beskos and Roberts (2005) and Beskos et al. (2006), the methodology is now much more advanced and includes: probabilistic results on the absolute maximum of Brownian paths and the maximum of Bessel process paths; a novel rejection sampling algorithm for the simulation of Brownian paths with known range. In the cited papers, the methodology required only a known result for the decomposition of a Brownian path at its minimum.

The paper is organised as follows. In Section 2 we introduce the diffusion law factorization which gives rise to the explicit diffusion construction, and we provide two classes of processes for which this factorization has already been used for their exact simulation in Beskos et al. (2006). Section 3 provides a general construction for one-dimensional homogeneous diffusions made possible under the new methodology presented in this paper. This is generalised in Section 4 to multivariate and time-inhomogeneous diffusions. Section 5 gives an explicit construction for the hitting time of Brownian motion to an arbitrary smooth boundary. This construction extends naturally to non-linear diffusions. Section 6 concludes with some remarks about possible extensions and future work.

\section{The Wiener-Poisson factorisation of $\mathbb{Q}$}

Additionally to $\left(\mathrm{C}_{0}\right)-\left(\mathrm{C}_{2}\right)$, we assume that the following condition holds for the rest of the paper:

$\left(\mathrm{C}_{3}\right)$ The function $h(u)=\exp \left\{-(u-x)^{2} /(2 t)+A(u)\right\}$ is integrable.

This is a weak assumption, since for example it is satisfied (at least for sufficiently small $t$ ) when a linear growth bound is assumed for the drift. Then, we define the probability measure $\mathbb{Z}$ through its Radon-Nikodym derivative with respect to $\mathbb{W}$ :

$$
\frac{\mathrm{d} \mathbb{Z}}{\mathrm{d} \mathbb{W}} \propto \exp \left\{A\left(X_{t}\right)-A(x)\right\} .
$$

Note that conditionally on the ending point $X_{t}$ the two laws coincide. So to simulate paths from $\mathbb{Z}$ one should draw first the ending point $X_{t} \sim h$ and then use Brownian bridge dynamics for the rest of the path. 
Let $\mathbb{L}$ denote the law of a unit rate Poisson process on $[0, t] \times[0, \infty)$ (see Kingman, 1993, for a formal definition) and define the extended law $\mathbb{Z} \otimes \mathbb{L}$ with typical realisation $(X, \Phi)$. When necessary, we write $\Phi=\left\{\left(\chi_{j}, \psi_{j}\right)\right\}_{j \geq 1}$, with $\left\{\psi_{j}\right\}$ non-decreasing. We define the non-negative drift functional,

$$
\phi(u):=\frac{\alpha^{2}(u)+\alpha^{\prime}(u)}{2}-\ell, \text { where } \ell=\inf _{z \in \mathbf{R}} \frac{\alpha^{2}(z)+\alpha^{\prime}(z)}{2},
$$

and consider the epigraph of $s \mapsto \phi\left(X_{s}\right)$,

$$
\text { epi } \phi(X):=\left\{(s, u) \in[0, t] \times \mathbf{R}_{+}: \phi\left(X_{s}\right) \leq u\right\},
$$

and the event

$$
\Gamma:=\{\Phi \subset \operatorname{epi} \phi(X)\}=\bigcap_{j \geq 1}\left\{\phi\left(X_{\chi_{j}}\right)<\psi_{j}\right\} .
$$

Theorem 1 (Wiener-Poisson factorization). $\mathbb{Q}$ is the marginal distribution of $X$ when $(X, \Phi) \sim \mathbb{Z} \otimes \mathbb{L} \mid \Gamma$

Proof. (2) and (3) yield that

$$
\frac{\mathrm{d} \mathbb{Q}}{\mathrm{d} \mathbb{Z}} \propto \exp \left\{-\int_{0}^{t} \phi\left(X_{s}\right) \mathrm{d} s\right\}
$$

From the explicit calculation of the zero probability of a Poisson random variable:

$$
\mathbb{L}(\Gamma \mid X)=\exp \left\{-\int_{0}^{t} \phi\left(X_{s}\right) \mathrm{d} s\right\} .
$$

Thus the result holds by Bayes's theorem.

Theorem 1 suggests a rejection sampling for the simulation of sample paths from $\mathbb{Q}$ : propose $X$ from $\mathbb{Z}$, simulate $\Phi$, and if $\Gamma$ has occurred then return $X$. This algorithm can be seen as a construction on the space $C[0, t] \times([0, t] \times[0, \infty))^{\mathbf{N}}$. However, the aim of this paper is to provide exact but finite constructions of $X$. This can be done, under the following general scheme. We construct a finite-dimensional random variable $\Upsilon$, and a positive function $r$ with the properties that:

1. $r(\Upsilon)<\infty$ a.s., 
2. $\sup _{s \in[0, t]} \phi\left(X_{s}\right) \leq r(\Upsilon)$ a.s..

In Section 5 we will allow $\Upsilon$ to be constructed on an enlargement of the canonical probability space for $X$. However, for immediate application we shall just use $\Upsilon=\Upsilon(X)$. Hence, under the scenario described in 1., 2. above, the event $\Gamma$ in (4) occurs if and only if the points of $\Phi$ which lie in the domain $[0, t] \times[0, r(\Upsilon)]$ are also in the epigraph of $s \mapsto \phi\left(X_{s}\right)$. That is

$$
\Gamma \equiv \Gamma_{r(\Upsilon)}:=\bigcap_{j: \psi_{j}<r(\Upsilon)}\left\{\phi\left(X_{\chi_{j}}\right)<\psi_{j}\right\}
$$

We define

$$
\Phi_{r(\Upsilon)}=\Phi \cap([0, t] \times[0, r(\Upsilon)])
$$

which is easy to construct as a Poisson process on $[0, t] \times[0, r(\Upsilon)]$.

\section{Exact Algorithm (EA)}

1. Simulate $X_{t} \sim h$.

2. Simulate $\Upsilon$ conditionally on $X_{t}$.

3. Simulate $\Phi_{r(\Upsilon)}$.

4. Simulate $\left.\left\{X_{\chi_{i}} ; 1 \leq i \leq\left|\Phi_{r(\Upsilon)}\right|\right\}\right\}$ from $\mathbb{Z} \mid X_{t}, \Upsilon$.

5. If $\Gamma_{r(\Upsilon)}$ has occurred output $\left.\left\{X_{\chi_{i}} ; 1 \leq i \leq\left|\Phi_{r(\Upsilon)}\right|\right\}\right\}$, otherwise goto 1 .

When $\Gamma_{r(\Upsilon)}$ has occurred, $\left\{X_{\chi_{i}} ; 1 \leq i \leq\left|\Phi_{r(\Upsilon)}\right|\right\} \sim \mathbb{Q}$. Thus, the only remaining challenge is to characterise the conditional distributions at steps 2 . and 4 . of the algorithm. The two steps are carried out conditionally on the ending point $X_{t}$. From the definition of $\mathbb{Z}$, this implies that we will actually work with Brownian bridge dynamics. In the rest of this Section we describe two special cases of EA already presented in Beskos et al. (2006). We present novel applications of EA in the sections that follow. 


\subsection{Exact Algorithm 1 (EA1)}

In Beskos et al. (2006) the Exact Algorithm (EA) was developed under the strong condition

$(* *)$ the function $\phi$ is bounded above.

In this simplified setting, additional information about the proposed path is not required to determine the restricted Poisson process, since it suffices to take

$$
r(u) \equiv r=\sup _{z \in \mathbf{R}} \phi(z)
$$

Thus, step 4. merely involves Brownian bridge simulation. We define the skeleton

$$
\mathcal{S}=\left\{\left(0, X_{0}\right),\left(t, X_{t}\right)\right\} \cup\left\{\left(\chi_{i}, X_{\chi_{i}}\right) ; 1 \leq i \leq\left|\Phi_{r}\right|\right\}
$$

An important feature of the method, stated in Theorem 2 below, is that the distribution of $X \mid \mathcal{S}, \Gamma$ is derived solely from the proposal measure $\mathbb{Z}$. Let $\mathbb{B} \mathbb{B}\left(s_{1}, s_{2} ; u_{1}, u_{2}\right)$ denote the law of a Brownian bridge starting at time $s_{1}$ at $u_{1}$ and ending at time $s_{2}$ at $u_{2}$. When $s_{1}=0$ we write $\mathbb{B B}\left(s_{2} ; u_{1}, u_{2}\right)$; we simplify the notation to $\mathbb{B B}$ when the parameters specifying the bridge are clearly understood from the context.

Theorem 2. Under $\left(C_{0}\right)-\left(C_{3}\right)$ and $\left(^{* *}\right)$,

$$
X \mid \mathcal{S}, \Gamma \sim \bigotimes_{i=1}^{\left|\Phi_{r}\right|+1} \mathbb{B} \mathbb{B}\left(s_{i-1}, s_{i} ; X_{s_{i-1}}, X_{s_{i}}\right)
$$

where $\left\{s_{i} ; 0 \leq i \leq\left|\Phi_{r}\right|+1\right\}$ is an increasing ordering of the set $\{0, t\} \cup\left\{\chi_{i} ; 1 \leq i \leq\left|\Phi_{r}\right|\right\}$.

Proof. Notice that $X|\mathcal{S}, \Gamma \stackrel{d}{=} X| \mathcal{S}$.

\subsection{EA2 and decomposition of the Brownian path at its minimum}

Beskos et al. (2006) replaced condition $\left(^{* *}\right)$ with the weaker $\left(^{*}\right)$. Without loss of generality, assume that $\limsup _{u \rightarrow \infty}\left(\alpha^{2}+\alpha^{\prime}\right)(u)<\infty$, so the function $\phi$ is bounded above on $[u, \infty)$ for any real $u$. Let $m=\inf \left\{X_{s} ; s \in[0, t]\right\}$. It suffices to set

$$
\Upsilon=m, \quad \text { and } \quad r(u)=\sup _{z \in[u, \infty)} \phi(z), \quad u \in \mathbf{R} .
$$


The law of the minimum of a Brownian bridge is known (Shepp, 1979) and it can be easily simulated using the inverse CDF method (see Proposition 2 of Beskos et al., 2006). Step 4. of EA requires the simulation of a Brownian bridge with a given minimum. This conditioned process can be represented in terms of two independent Bessel bridges (see for example Asmussen et al., 1995). The construction proceeds as follows.

We will need the instance of the minimum $\tau=\sup \left\{s \in(0, t): X_{s}=m\right\}$. We denote by $\mathbb{S}\left(s_{1}, s_{2} ; u_{1}, u_{2}\right)$ the law of a (3-dimensional) Bessel bridge starting at time $s_{1}$ at $u_{1} \geq 0$ and finishing at time $s_{2}$ at $u_{2} \geq 0$. We simplify to $\mathbb{S}\left(s_{2} ; u_{1}, u_{2}\right)$ when $s_{1}=0$. Assume that $X_{t}=y$. Let $R_{1} \sim \mathbb{S}(\tau ; x-m, 0)$ and $R_{2} \sim \mathbb{S}(t-\tau ; 0, y-m)$ independently. The process

$$
m+R_{s, 1} \mathbb{I}[0 \leq s<\tau]+R_{s-\tau, 2} \mathbb{I}[\tau \leq s \leq t], \quad s \in[0, t],
$$

has law $\mathbb{B B}(t ; x, y)$. Simulation of $\tau$ conditionally on $m$ can be carried out following the algorithm given in Beskos et al. (2006). Also, Bessel bridges starting or finishing at 0 can be easily constructed in terms of three independent Brownian bridges. The explicit formula is stated in Section 5.

In this setting,

$$
\mathcal{S}=\left\{\left(0, X_{0}\right),\left(t, X_{t}\right),\left(\tau, X_{\tau}\right)\right\} \cup\left\{\left(\chi_{i}, X_{\chi_{i}}\right) ; 1 \leq i \leq\left|\Phi_{r(\Upsilon)}\right|\right\}
$$

As in EA1, the conditional law of $X$ given all simulated variables is easily identified.

Theorem 3. Under $\left(C_{0}\right)-\left(C_{3}\right)$, and $\left(^{*}\right)$,

$$
X-\Upsilon \mid \mathcal{S}, \Upsilon, \Gamma \sim \bigotimes_{i=1}^{\left|\Phi_{r(\Upsilon)}\right|+2} \mathbb{S}\left(s_{i-1}, s_{i} ; X_{s_{i-1}}-\Upsilon, X_{s_{i}}-\Upsilon\right)
$$

where $\left\{s_{i} ; 0 \leq i \leq\left|\Phi_{r(\Upsilon)}\right|+2\right\}$ is an increasing ordering of $\{0, t, \tau\} \cup\left\{\chi_{i} ; 1 \leq i \leq\left|\Phi_{r(\Upsilon)}\right|\right\}$.

\section{$3 \quad$ Exact Algorithm 3 (EA3)}

In this section we demonstrate how to apply EA when only $\left(\mathrm{C}_{0}\right)-\left(\mathrm{C}_{3}\right)$ are assumed. Thus, we now make no upper bound assumptions on the function $\phi$. Recall that the random element $\Upsilon=\Upsilon(X)$ should be selected in way that $\sup _{s \in[0, t]} \phi\left(X_{s}\right) \leq r(\Upsilon)<\infty$ so it should 
now contain information for the whole range of the path $X$, not just the minimum as for EA2. We will define $\Upsilon$ as the label to an appropriate partition of the path space. Each set in the partition contains paths that move within a known interval. Thus, conditionally on $\Upsilon=\Upsilon(X)$ we will be in position to obtain a superset of $[\min (X), \max (X)]$ and, then, the required upper bound on $s \mapsto \phi\left(X_{s}\right)$. Recall that the construction should respect steps 2 . and 4. of EA, that is allow for the simulation of $\Upsilon$ and, then, of $X$ given $\Upsilon$ at any given time instances (of finite number).

We begin with the definition of the above-mentioned partition of the path space. Let $\left\{a_{i}\right\}_{i \geq 1}$ and $\left\{b_{i}\right\}_{i \geq 1}$ be two increasing sequence of positive real numbers, and $a_{0}=b_{0}=0$. Given $X_{0}=x, X_{t}=y$, we set $\bar{x}=x \wedge y, \bar{y}=x \vee y$, and define the following events:

$$
\begin{aligned}
& U_{i}=\left\{\sup _{0 \leq s \leq t} X_{s} \in\left[\bar{y}+b_{i-1}, \bar{y}+b_{i}\right)\right\} \cap\left\{\inf _{0 \leq s \leq t} X_{s}>\bar{x}-a_{i}\right\}, \\
& L_{i}=\left\{\inf _{0 \leq s \leq t} X_{s} \in\left(\bar{x}-a_{i}, \bar{x}-a_{i-1}\right]\right\} \cap\left\{\sup _{0 \leq s \leq t} X_{s}<\bar{y}+b_{i}\right\},
\end{aligned}
$$

and

$$
D_{i}=U_{i} \cup L_{i}, i \geq 1
$$

The required partition of the sample space consists of the sets $D_{i}, i \geq 1$. We introduce the discrete random variable $I=I(X)$ such that $\{I=i\}=D_{i}$. Note that $\{I \leq i\}$ is equivalent to $\left\{\bar{x}-a_{i}<X_{s}<\bar{y}+b_{i}\right.$, for all $\left.s \in[0, t]\right\}$. Thus, we set

$$
\Upsilon=I, \quad \text { and } \quad r(i)=\sup \left\{\phi(z) ; z \in\left(\bar{x}-a_{i}, \bar{y}+b_{i}\right)\right\}, \quad i \in \mathbf{N}
$$

Figure 1 demonstrates the construction.

In the remainder of the section we exploit a representation of the CDF of $I$ in terms of monotone alternating series, to develop a novel algorithm for its simulation. We then show how to simulate the Brownian bridge conditioned on $I$. In the sequel, we will sometimes call $I$ the layer of the Brownian bridge. Accordingly, we refer to a Brownian bridge conditioned on some value of $I$ as layered Brownian bridge. The algorithm devised for the simulation of $I$ can be readily modified to develop a rejection sampler of the layered Brownian bridge using Brownian bridge proposals. However, the number of proposed paths until the first 


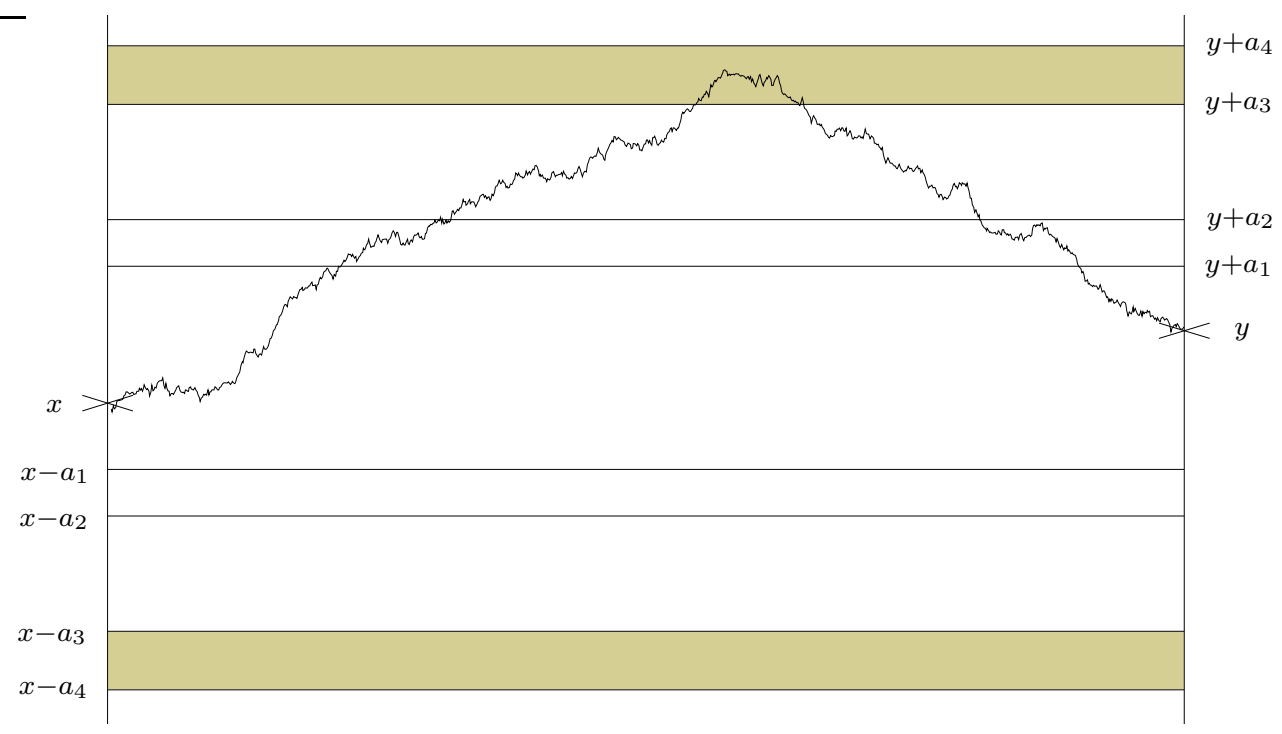

Figure 1: A sample path from $X_{0}=x$ to $X_{t}=y$, with $x<y$. In this example we have used symmetric layers, $a_{i}=b_{i}, i \geq 1$. For the specific choice of $\left\{a_{i}\right\}$ illustrated in the graph, the event $U_{4}$ has occurred. Thus, in this case $I(X)=4$.

acceptance has infinite expectation, where the expectation is taken w.r.t. proposed paths and $I$. This problem is alleviated by introducing an alternative rejection sampler for the simulation of the layered Brownian bridge.

\subsection{Some auxiliary results}

In the following subsections we identify events relevant to the construction of the layered Brownian bridge whose probability, $p$ say, is not known explicitly but it can be expressed as the limit of the following series of over/under-estimations:

$$
0<S_{2}<S_{4}<S_{6}<\cdots<p<\cdots<S_{5}<S_{3}<S_{1}
$$

for some explicitly known $\left\{S_{j}\right\}$. To simplify exposition we consider the following definition.

Definition 1. Let $\mathfrak{T}$ be the subset of Cauchy sequences with values in $\mathbf{R}_{+}$, such that $\left\{S_{j}\right\} \in \mathfrak{T}$ if and only if

$$
S_{2 j}<S_{2 j+2}<S_{2 j+1}<S_{2 j-1}, \quad \text { for all } j \geq 1 \text {. }
$$


For our constructions we will require simulation of events of probability $p=\lim _{j \rightarrow \infty} S_{j}$, where $\left\{S_{j}\right\} \in \mathfrak{T}$. Thus, we need to simulate the binary random variable $\mathbb{I}[U<p]$, where $U \sim \operatorname{Un}[0,1]$. This is accomplished in the following way. Let

$$
J=\inf \left\{j: j \text { even, } S_{j}>U \text { or } j \text { odd, } S_{j}<U\right\}
$$

Then

$$
\mathbb{I}[U<p]=\mathbb{I}[J \text { is even }] .
$$

Thus, one needs to consider only $J$ terms of $\left\{S_{j}\right\}$ to simulate the event of probability $p$. The specific alternating series that appear in our algorithms and are presented in the sequel, are converging in their limit faster than exponentially, so $J$ will be typically of a very small value.

In the sequel we will need to simulate events whose probabilities are linear transformations or ratios of alternating sequences in $\mathfrak{T}$. The following proposition illustrates that, for the case of these simple transformations, such probabilities can be also expressed as the limit of some easily identifiable sequences in $\mathfrak{T}$, therefore the corresponding events can be simulated as described above.

Proposition 1. Let $f: \mathbf{R}_{+}^{m} \rightarrow \mathbf{R}_{+}$, for some $m \in \mathbf{N}$, be a $C^{1}$ function with

$$
\left|\frac{\partial f}{\partial u_{i}}(\mathbf{u})\right|>0 \text { for all } 1 \leq i \leq m \text {, and } \mathbf{u} \in \mathbf{R}_{+}^{m} .
$$

Let $\left\{S_{j}^{i}\right\} \in \mathfrak{T}$, for all $1 \leq i \leq m$, with $p_{i}=\lim _{j \rightarrow \infty} S_{j}^{i}$, and set $p=f\left(p_{1}, \ldots, p_{m}\right)$. Consider the sequences $\left\{T_{j}^{i}\right\}$ defined for each $1 \leq i \leq m$ as follows:

$$
T^{i} \equiv S^{i} \text { if } \frac{\partial f}{\partial u_{i}}>0, \text { whereas } T_{.}^{i}=S_{\cdot+1}^{i} \quad \text { if } \frac{\partial f}{\partial u_{i}}<0 .
$$

Consider the sequence $\left\{S_{j}\right\}$ defined as follows:

$$
S_{j}=f\left(T_{j}^{1}, T_{j}^{2}, \ldots, T_{j}^{m}\right)
$$

Then $\left\{S_{j}\right\} \in \mathfrak{T}$ and $p=\lim _{j \rightarrow \infty} S_{j}$.

Proof. We need to prove that (6) holds for $\left\{S_{j}\right\}$ given that it holds for each of $\left\{S_{j}^{i}\right\}$. Such a conclusion is obvious when one considers the monotonicity structure of $f$. For the 
statement $S_{2 j+1}<S_{2 j-1}$, simply note that, when calculating $S_{2 j-1}, f$ takes as input higher values (compared to $S_{2 j+1}$ ) along the directions that $f$ is increasing and lower values along the directions that $f$ is decreasing. Similar considerations give the complete proof.

\subsubsection{The absolute maximum of a Brownian bridge}

For $K>\left|u_{1}\right| \vee\left|u_{2}\right|$, let $\gamma\left(s, u_{1}, u_{2}, K\right)$ denote the probability under $\mathbb{B} \mathbb{B}\left(s ; u_{1}, u_{2}\right)$ that a path does not leave the interval $[-K, K]$. This probability has a known expression in terms of an infinite series, a result which can be traced back to Doob (1949), although see Pötzelberger and Wang (2001) for a more recent reference. We define for $j \geq 1$,

$$
\begin{aligned}
& \bar{\sigma}_{j}\left(s, u_{1}, u_{2}, K\right)=\exp \left\{-\frac{2}{s}\left[2 K j-\left(K+u_{1}\right)\right]\left[2 K j-\left(K+u_{2}\right)\right]\right\}, \\
& \bar{\tau}_{j}\left(s, u_{1}, u_{2}, K\right)=\exp \left\{-\frac{2 j}{s}\left[4 K^{2} j+2 K\left(u_{1}-u_{2}\right)\right]\right\} .
\end{aligned}
$$

Then, Theorem 3 of Pötzelberger and Wang (2001) yields

$$
\gamma\left(s, u_{1}, u_{2}, K\right)=1-\sum_{j=1}^{\infty}\left\{\sigma_{j}\left(s, u_{1}, u_{2}, K\right)-\tau_{j}\left(s, u_{1}, u_{2}, K\right)\right\},
$$

where

$$
\sigma_{j}=\bar{\sigma}_{j}\left(s, u_{1}, u_{2}, K\right)+\bar{\sigma}_{j}\left(s,-u_{1},-u_{2}, K\right), \tau_{j}=\bar{\tau}_{j}\left(s, u_{1}, u_{2}, K\right)+\bar{\tau}_{j}\left(s,-u_{1},-u_{2}, K\right) .
$$

Notice that due to symmetry properties of the Brownian bridge, (7) can be used to compute the probability that an arbitrary Brownian bridge does not escape any given interval. Simulation of events of probability (7) can be achieved as described earlier due to Proposition 2 below.

Proposition 2. Let $\left\{S_{j}\right\}$ be constructed as follows:

$$
S_{2 j-1}=\sum_{k=1}^{j-1}\left(\sigma_{k}-\tau_{k}\right)+\sigma_{j}, S_{2 j}=S_{2 j-1}-\tau_{j}, \quad j \geq 1,
$$

where $\sigma_{j}=\sigma_{j}\left(s, u_{1}, u_{2}, K\right), \tau_{j}=\tau_{j}\left(s, u_{1}, u_{2}, K\right)$. Then $\left\{S_{j}\right\} \in \mathfrak{T}$ for any $u_{1}, u_{2} \in \mathbf{R}, s>0$, and $K>\left|u_{1}\right| \vee\left|u_{2}\right|$.

Proof. Routine calculation reveals that $\bar{\sigma}_{j}>\bar{\tau}_{j}>\bar{\sigma}_{j+1}$, for all $j \geq 1$. The result follows directly. 


\subsubsection{Hitting probabilities of the Bessel Bridge}

Consider some $u_{1} \geq 0, u_{2}>0$ and $K>u_{1} \vee u_{2}$. Let $\delta\left(s, u_{1}, u_{2}, K\right)$ denote the probability under the Bessel bridge law $\mathbb{S}\left(s ; u_{1}, u_{2}\right)$ that a path does not exceed $K$. Similarly, for $K<L$ we let $\delta\left(s, u_{1}, u_{2}, K ; L\right)$ denote the probability under $\mathbb{S}\left(s ; u_{1}, u_{2}\right)$ that a path conditioned to remain below $L$, does not exceed $K$. The derivation of these probabilities exploits the representation of a Bessel bridge as a Brownian bridge conditioned to stay positive. Thus, for $u_{1}>0$ and omitting the arguments of the Brownian bridge measure,

$$
\begin{gathered}
\delta\left(s, u_{1}, u_{2}, K ; L\right)=\frac{\mathbb{B B}[X \text { does not leave }(0, K)]}{\mathbb{B B}[X \text { does not leave }(0, L)]}=\frac{\gamma\left(s, u_{1}-K / 2, u_{2}-K / 2, K / 2\right)}{\gamma\left(s, u_{1}-L / 2, u_{2}-L / 2, L / 2\right)}, \\
\delta\left(s, u_{1}, u_{2}, K\right)=\lim _{L \rightarrow \infty} \delta\left(s, u_{1}, u_{2}, K ; L\right)=\frac{\gamma\left(s, u_{1}-K / 2, u_{2}-K / 2, K / 2\right)}{1-\exp \left\{-2 u_{1} u_{2} / s\right\}} .
\end{gathered}
$$

In this setting, $\mathbb{B} \mathbb{B} \equiv \mathbb{B B}\left(s ; u_{1}, u_{2}\right)$. For the special case $u_{1}=0$, we take the limit $u_{1} \rightarrow 0$ in the above expressions and find that,

$$
\begin{gathered}
\delta\left(s, 0, u_{2}, K ; L\right)=\frac{u_{2}-\sum_{j=1}^{\infty}\left\{\zeta_{j}\left(s, u_{2}, K\right)-\xi_{j}\left(s, u_{2}, K\right)\right\}}{u_{2}-\sum_{j=1}^{\infty}\left\{\zeta_{j}\left(s, u_{2}, L\right)-\xi_{j}\left(s, u_{2}, L\right)\right\}}, \\
\delta\left(s, 0, u_{2}, K\right)=1-\frac{1}{u_{2}} \sum_{j=1}^{\infty}\left\{\zeta_{j}\left(s, u_{2}, K\right)-\xi_{j}\left(s, u_{2}, K\right)\right\}
\end{gathered}
$$

where

$$
\xi_{j}\left(s, u_{2}, K\right)=\left(2 K j+u_{2}\right) \exp \left\{-2 K j\left(K j+u_{2}\right) / s\right\}, \zeta_{j}\left(s, u_{2}, K\right)=\xi_{j}\left(s,-u_{2}, K\right) .
$$

Simulation of events of probability $\delta\left(s, u_{1}, u_{2}, K ; L\right)$ and $\delta\left(s, u_{1}, u_{2}, K\right)$ can be easily achieved for any $u_{1}, u_{2}>0, L>K>u_{1} \vee u_{2}$, exploiting Proposition 1. For the limiting case, $u_{1}=0$, we additionally require the following result.

Proposition 3. Let $\left\{S_{j}\right\}$ be constructed as follows:

$$
S_{2 j-1}=\sum_{k=1}^{j-1}\left(\zeta_{k}-\xi_{k}\right)+\zeta_{j}, S_{2 j}=S_{2 j-1}-\xi_{j}, \quad j \geq 1,
$$

where $\zeta_{j}=\zeta_{j}\left(s, u_{2}, K\right), \xi_{j}=\xi_{j}\left(s, u_{2}, K\right)$, with $K>u_{2}>0$. If $3 K^{2}-s>0$, then $\left\{S_{j}\right\} \in \mathfrak{T}$. 
Proof. Notice that when $3 K^{2}-s>0$, then $f(u):=\zeta_{j}(s, u, K) / \xi_{j}(s, u, K)$ is increasing with $f(0)=1$. Thus, $\zeta_{j}>\xi_{j}$ for all appropriate values of their arguments. We will now show that $\xi_{j}>\zeta_{j+1}$. The ratio $\xi_{j} / \zeta_{j+1}$ is increasing in $j$ so it suffices to show that $\xi_{1}>\zeta_{2}$. Note that when $8 K^{2}-s>0$, then $g(u):=\xi_{1}(s, u, K) / \zeta_{2}(s, u, K)$ is decreasing with $g(K)=1$, thus $\xi_{1}>\zeta_{2}$. Having established the ordering $\zeta_{j}>\xi_{j}>\zeta_{j+1}$, the required result follows directly.

The restriction $3 K^{2}>s$ will not effect of general applicability of the algorithms that follow. As we show in the sequel, the user can easily specify an upper and lower bound on the possible range of values of $K$ and $s$ respectively so that it is guaranteed that $3 K^{2}>s$.

\subsection{Construction of layered Brownian bridge}

We return now to the setup we introduced at the beginning of Section 3. The distribution function of $I$ can be written as

$$
F(i):=\mathrm{P}[I \leq i]=\gamma\left(t, \frac{x-y}{2}+\frac{a_{i}-b_{i}}{2}, \frac{y-x}{2}+\frac{a_{i}-b_{i}}{2}, \frac{|y-x|}{2}+\frac{a_{i}+b_{i}}{2}\right), i \geq 1,
$$

with $F(0)=0$. Notice that $\mathrm{P}[I=i]=\mathrm{P}[F(i-1)<U \leq F(i)]$, for a $U \sim \operatorname{Un}[0,1]$, therefore $I$ can be simulated as follows. For any $i \geq 1$, let $\left\{S_{j}^{i}\right\} \in \mathfrak{T}$ be the alternating sequence converging to $F(i)$, as obtained from the representation of $\gamma$ in (7). We also define the sequence $\left\{S_{j}^{0}\right\}$ with $S_{j}^{0}=0$ for all $j \geq 1$. Then, we set $I=i$ when $S_{j}^{i-1}<U<S_{j+1}^{i}$, for some odd $j$.

Having simulated the layer $I$ of the Brownian bridge, we proceed to step 4. of EA, that is to simulate the resulted layered Brownian bridge at the finite collection of time instances determined by the Poisson process. The most obvious approach for doing so is to use rejection sampling with candidates from $\mathbb{B B}(t ; x, y)$ : for a proposed Brownian bridge path we simulate its layer and reject the path if this layer is different from the conditioned realisation of $I$. However this solution is theoretically and practically unappealing since if $N_{0}$ is the number of tries until (and including) the first success then:

$$
\mathrm{E}\left[N_{0}\right]=\mathrm{E}\left[\mathrm{E}\left[N_{0} \mid I\right]\right]=\sum_{i=1}^{\infty} \frac{1}{\mathrm{P}[I=i]} \times \mathrm{P}[I=i]=\infty .
$$


Therefore we adopt an alternative rejection sampling procedure which proposes sample paths which are more likely to be accepted. For the proposed construction we will consider symmetric layers, i.e. we choose $a_{i}=b_{i}, i \geq 1$. The construction could proceed even in the general case, however this user-specified tuning will simplify the formulae appearing in the sequel. Also, there is no obvious reason to give different weights to the upper and lower behaviour of the Brownian bridge.

Let $\mathbb{B B}_{D_{I}}$ denote the law of the target layered Brownian bridge. We will propose from a mixture of two conditioned Brownian bridges. Specifically, consider the events

$$
\overline{\mathrm{M}}_{i}=\left\{\sup _{0 \leq s \leq t} X_{s} \in\left[\bar{y}+a_{i-1}, \bar{y}+a_{i}\right)\right\}, \underline{\mathrm{M}}_{i}=\left\{\inf _{0 \leq s \leq t} X_{s} \in\left(\bar{x}-a_{i}, \bar{x}-a_{i-1}\right]\right\}, i \geq 1 .
$$

Let $\mathbb{B B B} \overline{\mathrm{M}}_{I}$ and $\mathbb{B B}_{\underline{\mathrm{M}}_{I}}$ be the Brownian bridge law $\mathbb{B B}(t ; x, y)$ restricted to $\overline{\mathrm{M}}_{I}$ and $\underline{\mathrm{M}}_{I}$ respectively. Our rejection sampling algorithm will use candidate paths from

$$
\mathbb{P}_{D_{I}}:=\frac{1}{2} \mathbb{B B}_{\overline{\mathrm{M}}_{I}}+\frac{1}{2} \mathbb{B B}_{\underline{\mathrm{M}}_{I}} .
$$

This proposal measure forces the minimum or the maximum of the realised paths to be in the correct interval, that is the interval where the maximum or the minimum of the target paths also lie. More analytically, recall the definition of the conditioning event $D_{I}$ from (5): $D_{I}=U_{I} \cup L_{I}$. The definition of the various events suggests that Brownian bridges from $\overline{\mathrm{M}}_{I}$ (resp. from $\underline{\mathrm{M}}_{I}$ ) are extremely good candidates for bridges from $U_{I}$ (resp. from $\left.L_{I}\right)$. The following theorem derives the appropriate acceptance probability. For a general subset of paths, say $\Delta, \mathbb{B} \mathbb{B}[\Delta]$ is the probability under $\mathbb{B} \mathbb{B}(t ; x, y)$ that a path is in $\Delta$.

Theorem 4. $\mathbb{B B}_{D_{I}}$ is absolutely continuous w.r.t. $\mathbb{P}_{D_{I}}$ with density

$$
\frac{\mathrm{d} \mathbb{B} \mathbb{B}_{D_{I}}}{\mathrm{dP}_{D_{I}}}(X)=\frac{2 \mathbb{B B}\left[\overline{\mathrm{M}}_{I}\right]}{\mathbb{B B}\left[D_{I}\right]} \times \frac{\mathbb{I}\left[X \in D_{I}\right]}{1+\mathbb{I}\left[X \in U_{I} \cap L_{I}\right]} .
$$

Proof. One only has to use the unconditioned Brownian bridge $\mathbb{B B}=\mathbb{B B}(t ; x, y)$ as a reference measure. Then:

$$
\begin{aligned}
\frac{\mathrm{d} \mathbb{B B}_{D_{I}}}{\mathrm{dP}_{D_{I}}}(X) & =\frac{\mathrm{d} \mathbb{B B} B_{D_{I}} / \mathrm{d} \mathbb{B B}(X)}{\frac{1}{2} \mathrm{~d} \mathbb{B B} \mathbb{M}_{I} / \mathrm{d} \mathbb{B B}(X)+\frac{1}{2} \mathrm{dBB}_{\underline{\mathrm{M}}_{I}} / \mathrm{d} \mathbb{B B B}(X)} \\
& =\frac{2 \mathbb{I}\left[X \in D_{I}\right] \mathbb{B B}\left[D_{I}\right]^{-1}}{\mathbb{I}\left[X \in \overline{\mathrm{M}}_{I}\right] \mathbb{B B B}\left[\overline{\mathrm{M}}_{I}\right]^{-1}+\mathbb{I}\left[X \in \underline{\mathrm{M}}_{I}\right] \mathbb{B B B}\left[\underline{\mathrm{M}}_{I}\right]^{-1}} .
\end{aligned}
$$


From the symmetricity of the layers it is clear that $\mathbb{B B}\left[\overline{\mathrm{M}}_{I}\right]=\mathbb{B B}\left[\underline{\mathrm{M}}_{I}\right]$, and the required density expression then follows directly.

Assume that $N$ is the number of proposed paths until the first accepted one. Then,

$$
\mathrm{E}[N \mid I]=\frac{2 \mathbb{B B}\left[\overline{\mathrm{M}}_{I}\right]}{\mathbb{B B B}\left[D_{I}\right]} \leq \frac{2 \mathbb{B B}\left[\overline{\mathrm{M}}_{I}\right]}{\mathbb{B B}\left[U_{I}\right]} \leq \frac{2}{\mathbb{B B}\left[\inf _{0 \leq s \leq t} X_{s}>\bar{x}-a_{1}\right]}
$$

so, even for moderate $a_{1}, \mathrm{E}[N]$ will be small. Note also that selecting symmetric layers $\left(a_{i}=b_{i}\right)$ simplifies the expression for the acceptance probability of the rejection sampler since, when that is the case, $\mathbb{B B B}\left[\overline{\mathrm{M}}_{I}\right]=\mathbb{B B B}\left[\underline{\mathrm{M}}_{I}\right]$; see the proof of the theorem above.

Our algorithm w.p. $1 / 2$ proposes a path $X \sim \mathbb{B B}_{\overline{\mathrm{M}}_{I}}$ and with equal probability a path $X \sim \mathbb{B B}_{\underline{\mathrm{M}}_{I}}$. Simulation from $\mathbb{B B}_{\underline{\mathrm{M}}_{I}}$ (and by symmetry from $\mathbb{B B}_{\overline{\mathrm{M}}_{I}}$ ) can be easily carried out following the decomposition of a Brownian bridge at its minimum in terms of two Bessel bridges summarized in Section 2.2. The calculation of the probability of accepting $X$ requires also the simulation of the indicators $\mathbb{I}\left[X \in D_{I}\right]$ and $\mathbb{I}\left[X \in U_{I} \cap L_{I}\right]$ which will be carried out using the results about the hitting times of a Bessel bridge given in Section 3.1.2. Analytically, we proceed as follows.

First, we simulate the minimum $m$ of the proposed Brownian bridge $X$ conditionally on that being in $\left(\bar{x}-a_{I}, \bar{x}-a_{I-1}\right]$, and then the time $\tau$ when the minimum is attained. Recall that the target layered path, thus also the proposed path, needs to be simulated only at the time instances of the Poisson process $\Phi_{r(\Upsilon)}$. In the simple case when $\Phi_{r(\Upsilon)}$ has no points, the binary variable $\mathbb{I}\left[X \in D_{I}\right]$ is easy to simulate, since it is equal to 1 w.p.

$$
\delta\left(\tau, 0, \bar{x}-m, \bar{y}+a_{I}-m\right) \times \delta\left(t-\tau, 0, \bar{y}-m, \bar{y}+a_{I}-m\right) .
$$

If $\mathbb{I}\left[X \in D_{I}\right]=0$ the path $X$ is rejected. In the opposite case, we proceed to the indicator $\mathbb{I}\left[X \in U_{I} \cap D_{I}\right]$. Conditionally on $\mathbb{I}\left[X \in D_{I}\right]=1$, it is true that $\mathbb{I}\left[X \in U_{I} \cap L_{I}\right]=0$, w.p.

$$
\delta\left(\tau, 0, \bar{x}-m, \bar{y}+a_{I-1}-m ; \bar{y}+a_{I}-m\right) \times \delta\left(t-\tau, 0, \bar{y}-m, \bar{y}+a_{I-1}-m ; \bar{y}+a_{I}-m\right) .
$$

If $\mathbb{I}\left[X \in U_{I} \cap L_{I}\right]=1$, then the proposed path is accepted w.p. 1/2, otherwise w.p. 1 .

In the general case when $\Phi_{r(\Upsilon)}$ has a positive number of points, the procedure is similar. We simulate, $m, \tau$, and the location of the proposed path at the time instances determined 
by $\Phi_{r(\Upsilon)}$. Conditionally on this information, the path is retrieved as a product of Bessel bridges. Thus, the distribution of the binary variables $\mathbb{I}\left[X \in D_{I}\right]$ and $\mathbb{I}\left[X \in U_{I} \cap L_{I}\right]$ can be expressed in terms of products of hitting probabilities of the Bessel bridge and can be simulated following the alternating series method shown in Section 3.1.

For more concreteness, we can formulate a pseudo-algorithm describing step 4. of EA3. We will need the maximum of a path $m^{*}:=\sup \left\{X_{s} ; s \in[0, t]\right\}$.

EA3 - Step 4. Simulation of $\left.\left\{X_{\chi_{i}} ; 1 \leq i \leq\left|\Phi_{r(\Upsilon)}\right|\right\}\right\}$ from $\mathbb{Z} \mid X_{t}=y, I$.

4.1 For $X \sim \mathbb{B} \mathbb{B}(t ; x, y)$, simulate $\left.\left\{X_{\chi_{i}} ; 1 \leq i \leq\left|\Phi_{r(\Upsilon)}\right|\right\}\right\}$ together with either it's minimum $(\tau, m)$, w.p. $1 / 2$, or it's maximum $\left(\tau, m^{*}\right)$, w.p. $1 / 2$, conditionally on $m \in\left(\bar{x}-a_{I}, \bar{x}-a_{I-1}\right]$ or $m^{*} \in\left[\bar{y}+a_{I-1}, \bar{y}+a_{I}\right)$.

4.2 If $\mathbb{I}\left[X \in D_{I}\right]=0$ reject $X$ and go to 4.1 .

4.4 If $\mathbb{I}\left[X \in U_{I} \cap L_{I}\right]=0$ (resp. 1) return $\left.\left\{X_{\chi_{i}} ; 1 \leq i \leq\left|\Phi_{r(\Upsilon)}\right|\right\}\right\}$ w.p. 1 (resp. 1/2). Otherwise reject $X$ and go to 4.1 .

For step 4.2, we note that, in the case that the minimum $m$ is simulated at step 4.2, $\mathbb{I}\left[X \in D_{I}\right]=1$ is an event of probability

$$
\prod_{i=1}^{\left|\Phi_{r(\Upsilon)}\right|+2} \delta\left(s_{i}-s_{i-1}, X_{s_{i-1}}-m, X_{s_{i}}-m, \bar{y}+a_{I}-m\right),
$$

where $\left\{s_{i} ; 0 \leq i \leq\left|\Phi_{r(\Upsilon)}\right|+2\right\}$ is an increasing ordering of $\{0, t, \tau\} \cup\left\{\chi_{i} ; 1 \leq i \leq\left|\Phi_{r(\Upsilon)}\right|\right\}$. For the case of the maximum $m^{*}$, one should only replace each of the terms in the above product with $\delta\left(s_{i}-s_{i-1}, m^{*}-X_{s_{i-1}}, m^{*}-X_{s_{i}}, m^{*}-\bar{x}+a_{I}\right)$. Then, for step 4.3 , the event $\mathbb{I}\left[X \in U_{I} \cap L_{I}\right]=0$ is of probability:

$$
\prod_{i=1}^{\left|\Phi_{r(\Upsilon)}\right|+2} \delta\left(s_{i}-s_{i-1}, X_{s_{i-1}}-m, X_{s_{i}}-m, \bar{y}+a_{I-1}-m ; \bar{y}+a_{I}-m\right)
$$

for the case of $m$, with $\delta\left(s_{i}-s_{i-1}, m^{*}-X_{s_{i-1}}, m^{*}-X_{s_{i}}, m^{*}-\bar{x}+a_{I-1} ; m^{*}-\bar{x}+a_{I}\right)$ replacing the terms in the above product for the case of $m^{*}$. 


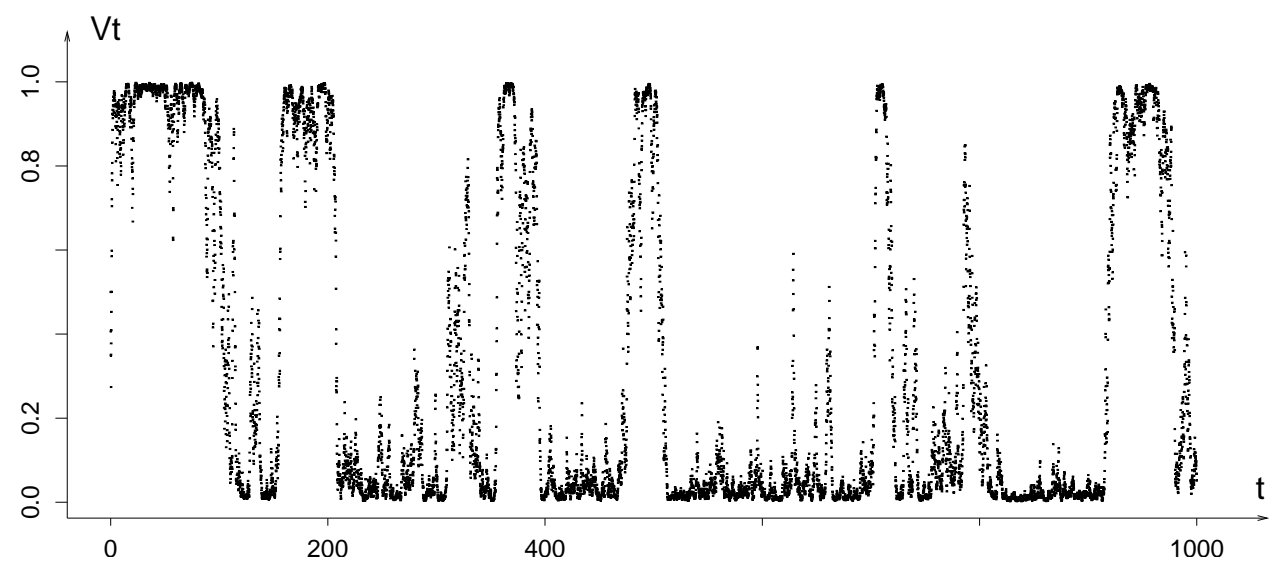

Figure 2: A skeleton of (8) generated by EA3. We used $\mu=0.01, \nu=-0.02, \sigma=1$ and starting point $V_{0}=0.5$. We applied iteratively EA3 for time increments $t=0.1$ up to the time 1000. The increasing sequences $\left\{a_{i}\right\},\left\{b_{i}\right\}$ involved in the algorithm were chosen as $a_{i}=b_{i}=0.3 \cdot i$.

\subsection{Examples}

The methodology we have developed applies easily to one-dimensional diffusions, $V$ say, with positive and continuously differentiable coefficient $\sigma(\cdot)$. We first apply the standard transformation: $V_{s} \mapsto \int_{v^{*}}^{V_{s}} \sigma(z)^{-1} \mathrm{~d} z=: X_{s}$, for some arbitrary $v^{*}$ in the state space of $V$, and then apply EA to $X$, provided that $\left(\mathrm{C}_{0}\right)-\left(\mathrm{C}_{3}\right)$ hold for $X$.

As an illustration of EA3, we consider the following diffusion model arising in genetics (see e.g. ch.7 of Kloeden and Platen, 1992):

$$
\mathrm{d} V_{s}=\left(\mu+\nu V_{s}\right) \mathrm{d} s+\sigma V_{s}\left(1-V_{s}\right) \mathrm{d} W_{s}
$$

with $\sigma>0$ and $\mu, \nu$ assumed to satisfy the restrictions $\mu>0$ and $\mu+\nu<0$ implying that $V$ stays in $(0,1)$. Under this parameter choice, straightforward calculations show that the corresponding unit coefficient process $X$, which moves in $\mathbf{R}$, satisfies conditions $\left(\mathrm{C}_{0}\right)-\left(\mathrm{C}_{3}\right)$. Thus, we can apply EA3 to simulate sample paths of $X$ and, equivalently, of $V$. Figure 2 shows a skeleton of $V$ simulated using EA3. 


\section{Extensions}

In this section we shall consider extensions of the results obtained and algorithms described thus far in the paper. We shall consider both multivariate and time-inhomogeneous generalizations.

\subsection{Multivariate diffusions}

We now consider unit diffusion coefficient $d$-dimensional diffusions satisfying

$$
\mathrm{d} \mathbf{X}_{s}=\boldsymbol{\alpha}\left(\mathbf{X}_{s}\right) \mathrm{d} s+\mathrm{d} \mathbf{B}_{s}, \quad X_{0}=\mathbf{x}, s \in[0, t]
$$

Let $\mathbb{Q}$ and $\mathbb{W}$ denote the law of $\mathbf{X}$ and the $d$-dimensional Wiener measure respectively on $[0, t]$ for the initial condition $\mathbf{X}_{0}=\mathbf{B}_{0}=\mathbf{x}$. We will assume the following multi-dimensional equivalents to $\left(\mathrm{C}_{0}\right)-\left(\mathrm{C}_{3}\right)$ :

$\left(\mathrm{MC}_{0}\right)$ The Radon-Nikodym derivative of $\mathbb{Q}$ w.r.t. $\mathbb{W}$ exists and it is given by the multivariate Girsanov's formula,

$$
\frac{\mathrm{d} \mathbb{Q}}{\mathrm{d} \mathbb{W}}=\exp \left\{\int_{0}^{t} \boldsymbol{\alpha}\left(\mathbf{X}_{s}\right) \cdot \mathrm{d} \mathbf{X}_{s}-\int_{0}^{t} \frac{1}{2}\left\|\boldsymbol{\alpha}\left(\mathbf{X}_{s}\right)\right\|^{2} \mathrm{~d} s\right\}
$$

$\left(\mathrm{MC}_{1}\right) \boldsymbol{\alpha}$ is continuously differentiable in all its arguments.

$\left(\mathrm{MC}_{2}\right)$ There exists $\ell>-\infty$ such that $\phi(\mathbf{u}):=\left(\|\boldsymbol{\alpha}(\mathbf{u})\|^{2}+\operatorname{div} \boldsymbol{\alpha}(\mathbf{u})\right) / 2-\ell \geq 0$.

Then, analogously to the one-dimensional case, we consider the the epigraph of $s \mapsto \phi\left(\mathbf{X}_{s}\right)$,

$$
\text { epi } \phi(\mathbf{X}):=\left\{(s, u) \in[0, t] \times \mathbf{R}_{+}: \phi\left(\mathbf{X}_{s}\right) \leq u\right\}
$$

and the event

$$
\Gamma:=\{\Phi \subset \text { epi } \phi(\mathbf{X})\}=\bigcap_{j \geq 1}\left\{\phi\left(\mathbf{X}_{\chi_{j}}\right)<\psi_{j}\right\}
$$

Theorem 1 extends readily to the multivariate case, however $\left(\mathrm{C}_{3}\right)$ has to be appropriately modified. Notice that $\left(\mathrm{MC}_{3.1}\right)$ below appears frequently in applications of multivariate diffusions.

$\left(\mathrm{MC}_{3.1}\right)$ There exists a function $A: \mathbf{R}^{d} \rightarrow \mathbf{R}$ such that $\boldsymbol{\alpha}(\mathbf{u})=\nabla A(\mathbf{u})$, 
$\left(\mathrm{MC}_{3.2}\right)$ The function $\exp \left\{A(\mathbf{u})-\|\mathbf{u}-\mathbf{x}\|^{2} /(2 t)\right\}$ is integrable in $\mathbf{u} \in \mathbf{R}^{d}$.

We then define the probability measure $\mathbb{Z}$ w.r.t. the $d$-dimensional Wiener measure as

$$
\frac{\mathrm{d} \mathbb{Z}}{\mathrm{d} \mathbb{W}} \propto \exp \left\{A\left(\mathbf{X}_{t}\right)-A(\mathbf{x})\right\}
$$

Theorem 5 (Multivariate Wiener-Poisson factorization). $\mathbb{Q}$ is the marginal law of $\mathbf{X}$ when $(\mathbf{X}, \Phi) \sim \mathbb{Z} \otimes \mathbb{L} \mid \Gamma$.

Theorem (5) leads directly to a simulation methodology as in the one-dimensional case. The most natural approach is to construct the proposed $d$-dimensional paths by means of $d$ independent Brownian bridges with some corresponding layer choice. However, the assumption of unit diffusion coefficient is not as innocuous as in the one-dimensional case. Unfortunately, there is no obvious way to generalize this given the currently available methodology.

\subsection{Time-inhomogeneous diffusions}

EA can also be applied to diffusion processes of the form:

$$
\mathrm{d} X_{s}=\alpha\left(s, X_{s}\right) \mathrm{d} s+\mathrm{d} B_{s}, \quad X_{0}=x, s \in[0, t] .
$$

The necessary conditions for the applicability of EA are straightforward restatements of $\left(\mathrm{C}_{0}\right)-\left(\mathrm{C}_{3}\right)$, thus we do not present them here explicitly. In the sequel, $\left(\mathrm{IC}_{0}\right)-\left(\mathrm{IC}_{3}\right)$ will stand for the extension of $\left(\mathrm{C}_{0}\right)-\left(\mathrm{C}_{3}\right)$ in the time-inhomogeneous context. In summary, we now ask that $\alpha \in C^{1,1}$, we define $A(s, u)=\int_{0}^{u} \alpha(s, z) \mathrm{d} z$, and we let $\mathbb{Z}$ be the law determined through the following density:

$$
\frac{\mathrm{d} \mathbb{Z}}{\mathrm{d} \mathbb{W}} \propto \exp \left\{A\left(t, X_{t}\right)\right\}
$$

Thus the Wiener-Poisson construction and its associated algorithms go through unchanged, setting

$$
\phi(s, u)=\frac{1}{2}\left\{\alpha^{2}(s, u)+\frac{\partial \alpha(s, u)}{\partial u}+2 \frac{\partial A(s, u)}{\partial s}\right\}-\ell
$$

where

$$
\ell=\inf _{s \in[0, t]} \inf _{z \in \mathbf{R}}\left\{\frac{1}{2} \alpha^{2}(s, z)+\frac{1}{2} \frac{\partial \alpha(s, z)}{\partial z}+\frac{\partial A(s, z)}{\partial s}\right\}
$$


Whereas $\left(\mathrm{C}_{2}\right)$ is a standard assumption, its extension to this setting, i.e. the condition that $\ell>-\infty$ for $\ell$ defined defined above, might not be easy to meet in great generality. A characteristic example is when $X$ is Brownian motion with time-varying drift, that is $X_{s}=B_{s}+f(s)$ for some smooth function $f$.

Simulation of time-inhomogeneous diffusions is an essential ingredient of the exact simulation algorithm of one-sided boundary hitting times that we present in the next section.

\section{Simulation of boundary hitting times}

In this section we recast the methodology we have already developed to devise an algorithm for the simulation of diffusion hitting times of one-sided smooth boundaries. These problems can be equivalently stated as constant boundary crossing times for timeinhomogeneous diffusions, hence the extension of EA3 described in Section 4.2 will be required. We will restrict ourselves to the important special case of Brownian motion and consider the problem of the exact simulation of the first time that the Brownian motion exceeds a non-linear boundary $f \in C^{2}$. Thus, we will present a method for the simulation of the random variable

$$
\zeta=\inf \left\{s \geq 0: B_{s}=f(s)\right\}
$$

where $B$ is a standard Brownian motion. Without loss of generality we will assume that $f(0)>0$.

An attempt to simulate the time-inhomogeneous process $B_{s}-f(s)$ using EA with the objective to exploit the availability of results for crossing times of linear boundaries for the Brownian motion would fail since $\left(\mathrm{IC}_{2}\right)$ does not hold. Notice that there are more general diffusion processes for which this inconvenience will not appear. We get around this difficulty by re-expressing the problem in terms of an Ornstein-Uhlenbeck process.

For an arbitrary tuning constant $a \in \mathbf{R}_{+}$, we consider the process

$$
H_{s}:=e^{-a s / 2} B_{\left(e^{a s}-1\right) / a},
$$

which satisfies $\mathrm{d} H_{s}=-(a / 2) H_{s} \mathrm{~d} s+\mathrm{d} W_{s}, H_{0}=0$, for a suitable Brownian motion $W$. 
We define the modified boundary

$$
g(s)=e^{-a s / 2} f\left(\left(e^{a s}-1\right) / a\right)
$$

and consider the hitting time

$$
\eta=\inf \left\{s \geq 0: H_{s}=g(s)\right\}
$$

It is clear that $\zeta=\left(e^{a \eta}-1\right) / a$ a.s., thus is suffices to simulate $\eta$. We define the timeinhomogeneous diffusion $X_{s}:=g(s)-H_{s}$ which satisfies

$$
\mathrm{d} X_{s}=\left(g^{\prime}(s)+a g(s) / 2-a X_{s} / 2\right) \mathrm{d} s+\mathrm{d} W_{s}, \quad X_{0}=g(0)>0 .
$$

Now, $\eta$ is the first passage time of $X$ from 0 .

We will simulate $\eta$ through rejection sampling on the path space, using a variation of EA3 for time-inhomogeneous diffusions. The target diffusion will be $X$ defined above. It can be easily shown that conditions $\mathrm{IC}_{0}-\mathrm{IC}_{3}$ are satisfied. The algorithm will exploit the fact that the crossing times of linear boundaries of the proposed Brownian bridges are explicitly known and can be easily simulated.

We begin with the following well-known first passage time decomposition of $\mathbb{B B}(t ; x, y)$. Recall the definitions of $m, \tau$ and $\mathbb{S}$ given in Section 2.2 as the minimum of a path, the time that the minimum occurs, and the law of a Bessel bridge, respectively.

Theorem 6. Assume that $x>0$ and let $\eta$ denote the first time the bridge $X \sim \mathbb{B B}(t ; x, y)$ hits 0 , where by convention we set $\eta=t$ if the hitting time does not occur in $[0, t]$.

1. For $\eta<t$, the law of $X \mid \eta$ admits the decomposition

$$
\mathbb{S}(\eta ; x, 0) \otimes \mathbb{B B}(\eta, t ; 0, y)
$$

2. For $\eta=t$, the law of $X-m \mid \eta=t, m, \tau$ can be decomposed as

$$
\mathbb{S}(\tau ; x-m, 0) \otimes \mathbb{S}(\tau, t ; 0, y-m)
$$

Therefore, the additional conditioning on the hitting time $\eta$ has the effect that the proposed paths on $[0, \eta]$ are now expressed in terms of Bessel bridges. Although simulation of $I$ is 
still possible (using the Bessel bridge hitting probabilities of Section 3.1.2), the algorithm of Section 3.2 for simulating the layered path cannot be applied, since that construction only applies to Brownian bridges.

We will nonetheless obtain lower and upper bounds for the proposed path by exploiting the well-known representation (see for instance Bertoin and Pitman, 1994) of the Bessel bridge starting from 0 , in terms of three independent Brownian bridges. Analytically, for some $\delta>0$ and a time increment $v>0$, the stochastic process

$$
\sqrt{Z_{s, 1}^{2}+Z_{s, 2}^{2}+Z_{s, 3}^{2}}, s \in[0, v], \text { where } Z_{1} \sim \mathbb{B B}(v ; 0, \delta), Z_{2}, Z_{3} \sim \mathbb{B B}(v ; 0,0)
$$

is distributed according to $\mathbb{S}(v ; 0, \delta)$. All Bessel bridges appearing in Theorem 6 are covered by this result due to the time-homogeneity of the Bessel bridge and its time-reversing property.

Our approach is to determine separate layers for each of the Brownian bridges that constitute the proposed Bessel bridge, using the techniques of Section 3. These layers will imply a bound on the range of the Bessel bridge. Simulation of the Bessel bridge conditional on this information will be done via the simulation of each constituent Brownian bridge conditional on its layer using the efficient rejection sampler of Section 3.2.

The precise mathematical construction is as follows. We will consider ten independent Brownian bridges. The first four

$$
Z_{1} \sim \mathbb{B B}(\eta ; x, 0), Z_{2}, Z_{3} \sim \mathbb{B B}(\eta ; 0,0), Z \sim \mathbb{B B}(\eta, t ; 0, y)
$$

will be used in the case $\eta<t$, and the rest

$$
\begin{gathered}
Z_{5} \sim \mathbb{B B}(\tau ; x-m, 0), Z_{6}, Z_{7} \sim \mathbb{B B}(\tau ; 0,0), \\
Z_{8} \sim \mathbb{B B}(\tau, t ; 0, y-m), Z_{9}, Z_{10} \sim \mathbb{B B}(\tau, t ; 0,0),
\end{gathered}
$$

when $\eta=t$. For each bridge $Z_{i}$, we define $\bar{x}_{i}\left(\bar{y}_{i}\right)$ to be the minimum (maximum) between its starting and finishing point, and provide a positive increasing sequence $\left\{a_{j}\right\}$ for the development of symmetric layers around $\bar{x}_{i}$ and $\bar{y}_{i}$ in the way shown in Section 3. For simplicity, we assume the choice of an identical sequence $\left\{a_{j}\right\}$ for all ten bridges. In greater 
generality, one can select a different sequence for each bridge. Let $I_{i}$ be the discrete random variable which specifies the layer of $Z_{i}$ and $\mathbf{I}=\left\{I_{i} ; 1 \leq i \leq 10\right\}$. In this setting, $\Upsilon$ is defined on an enlargement of the canonical probability space for $X$, as

$$
\Upsilon=(\eta, \mathbf{I}, m \mathbb{I}[\eta=t], \tau \mathbb{I}[\eta=t])
$$

Then, the proposed Brownian bridge and a bound on its range are constructed as follows.

- If $\eta<t$, then

$$
X_{s}=\sqrt{Z_{s, 1}^{2}+Z_{s, 2}^{2}+Z_{s, 3}^{2}}, s \in[0, \eta), \quad X_{s}=Z_{s, 4}, s \in[\eta, t],
$$

and $l(\mathbf{I}) \leq X_{x} \leq u(\mathbf{I})$ for all $s \in[0, t]$, where

$$
l(\mathbf{I})=\bar{x}_{4}-a_{I_{4}}, \quad u(\mathbf{I})=\max \left\{\sqrt{\left(x+a_{I_{1}}\right)^{2}+a_{I_{2}}^{2}+a_{I_{3}}^{2}}, \bar{y}_{4}+a_{I_{4}}\right\}
$$

- If $\eta=t$, then

$$
X_{s}=m+\sqrt{Z_{s, 5}^{2}+Z_{s, 6}^{2}+Z_{s, 7}^{2}}, s \in[0, \tau) ; X_{s}=m+\sqrt{Z_{s, 8}^{2}+Z_{s, 9}^{2}+Z_{s, 10}^{2}}, s \in[\tau, t],
$$

and $l(\mathbf{I}) \leq X_{s} \leq u(\mathbf{I})$ for all $0 \leq s \leq t$, where $l(\mathbf{I})=m$ and

$$
u(\mathbf{I})=m+\max \left\{\sqrt{\left(x-m+a_{I_{5}}\right)^{2}+a_{I_{6}}^{2}+a_{I_{7}}^{2}}, \sqrt{\left(y-m+a_{I_{8}}\right)^{2}+a_{I_{9}}^{2}+a_{I_{10}}^{2}}\right\} .
$$

Given $l(\mathbf{I}), u(\mathbf{I})$ we can compute the rate of the auxiliary Poisson process as

$$
r(\Upsilon)=\sup \{\phi(s, z): s \in[0, t], z \in[l(\mathbf{I}), u(\mathbf{I})]\}
$$

and, subsequently, complete the program prescribed in EA to decide whether or not to accept the proposed path and the corresponding hitting time. Notice, that in practice it is not necessary to find apriori ranges for all ten bridges since the specific bridges required depend on the value of $\eta$.

Hitting times of two sided boundaries are in general not possible by this approach. 


\section{Discussion}

In this paper we have reconstructed the law of a family of diffusions in terms of the Wiener and the Poisson measures. Based on this representation, we devised a simulation algorithm for un-solvable SDEs. Our algorithm, EA3, is described in Section 3 as an explicit finite construction for exact realisations from the finite dimensional distributions of the diffusion law. The methodology extends easily to time-inhomogeneous and multivariate diffusions, though the latter generalization is restricted to diffusions with gradient drift functions.

Crucial to our approach is the layered Brownian bridge construction which we initially describe in the EA3 context. In Section 5 we show that the same device used in a modified way can generate realisations of boundary crossing times for diffusions. The layered Brownian bridge construction is rather versatile. For instance it is straightforward to construct diffusion sample paths conditioned to remain within a region, $E$, (compact or otherwise) by just arranging that the initial layer $(I=1)$ corresponds to the sample path remaining within $E$. Furthermore diffusions with finite entrance boundaries such as

$$
\mathrm{d} X_{t}=-\tan \left(X_{t}\right) \mathrm{d} t+\mathrm{d} B_{t}
$$

can be easily simulated by choosing sequences of layers with edges converging to $\pm \pi / 2$.

A close inspection of the EA3 construction reveals that it allows the condition $\left(\mathrm{C}_{0}\right)$ to be relaxed. The algorithm certainly requires the diffusion to be non-explosive. However the layered construction only requires the Radon-Nikodym derivative of $\mathbb{Q}$ w.r.t. $\mathbb{W}$ to exist individually on each layer event $\{I \leq i\}$. By $\left(\mathrm{C}_{1}\right)$, the path $s \mapsto \alpha\left(X_{s}\right)$ is bounded on the event $\{I \leq i\}$ and so Girsanov's formula holds on each of these truncations. Thus we can relax $\left(\mathrm{C}_{0}\right)$ to the statement that

$$
\left(\mathrm{C}_{0}\right)^{\prime} X \text { is a non-explosive diffusion. }
$$

\section{Acknowledgements}

The authors would like to thank Bruno Casella for several helpful discussions on the subjects of this paper. They also gratefully acknowledge support from EPSRC grant GR/S61577/01. 
One of the authors research was funded by the Greek State Scholarship's Foundation.

\section{References}

Asmussen, S., Glynn, P. and Pitman, J. (1995) Discretization error in simulation of onedimensional reflecting Brownian motion. Ann. Appl. Probab., 5, 875-896.

Bertoin, J. and Pitman, J. (1994) Path transformations connecting Brownian bridge, excursion and meander. Bull. Sci. Math., 118, 147-166.

Beskos, A., Papaspiliopoulos, O. and Roberts, G. O. (2006) Retrospective exact simulation of diffusion sample paths with applications. Bernoulli, 12, 1077-1098.

Beskos, A. and Roberts, G. O. (2005) Exact simulation of diffusions. Ann. Appl. Probab., 15, 2422-2444.

Doob, J. L. (1949) Heuristic approach to the Kolmogorov-Smirnov theorems. Ann. Math. Statistics, 20, 393-403.

Kingman, J. F. C. (1993) Poisson processes, vol. 3 of Oxford Studies in Probability. New York: The Clarendon Press Oxford University Press. ,Oxford Science Publications.

Kloeden, P. E. and Platen, E. (1992) Numerical solution of stochastic differential equations, vol. 23 of Applications of Mathematics (New York). Berlin: Springer-Verlag.

Pötzelberger, K. and Wang, L. (2001) Boundary crossing probability for Brownian motion. J. Appl. Probab., 38, 152-164.

Shepp, L. A. (1979) The joint density of the maximum and its location for a Wiener process with drift. J. Appl. Probab., 16, 423-427. 